\title{
Formation postgraduée et continue: un continuum
}

\begin{abstract}
L'ISFM a-t-il eu des défis exceptionnels à relever en 2017? Christoph Hänggeli: Et comment! Le plus grand défi que nous avons eu à relever a été la restructuration du logbook électronique. La version actuelle remplit encore ses fonctions pour documenter la formation accomplie mais elle ne peut plus évoluer de manière pertinente et sa maintenance est très coûteuse. Pour nos informaticiens, l'architecture logicielle est insuffisante par rapport aux possibilités actuelles et ne permet pas de changements importants au niveau du catalogue des objectifs de formation. Pour l'exprimer simplement, il est tout à fait possible par exemple qu'un objectif de formation soit supprimé en psychiatrie quand une nouvelle intervention doit être ajoutée en urologie. Nous devons avoir cette marge.
\end{abstract}

\section{Et maintenant?}

Christoph Hänggeli: Tous les analystes sont arrivés à la même conclusion. Il serait beaucoup plus rapide et meilleur marché de repartir sur une nouvelle base avec un logbook électronique 2.0 au lieu de réparer le sys- tème en place. Les experts nous ont garanti que la nouvelle version modulaire disposait d'une programmation beaucoup mieux structurée qui pourra répondre entièrement aux exigences futures. Dans un premier temps, cela signifie des coûts importants, mais l'ISFM peut les assumer grâce à ses réserves pécuniaires. En revanche, nous devrons serrer un peu plus les cordons de la bourse pendant quelques années et mettre d'autres projets en attente.

Giatgen Spinas: J'aimerais ajouter que le logbook électronique occupe une place centrale pour les médecins en formation et qu'il doit donc fonctionner sans problème. A l'avenir, il permettra aussi de traiter plus rapidement les demandes de titres, de fournir des informations statistiques importantes, notamment pour le financement des postes de formation par les cantons.

\section{L'année 2017 a-t-elle aussi été porteuse de bonnes nouvelles?}

Werner Bauer: Fondamentalement, le modèle de for mation postgraduée suisse a du succès, c'est ce qui res-

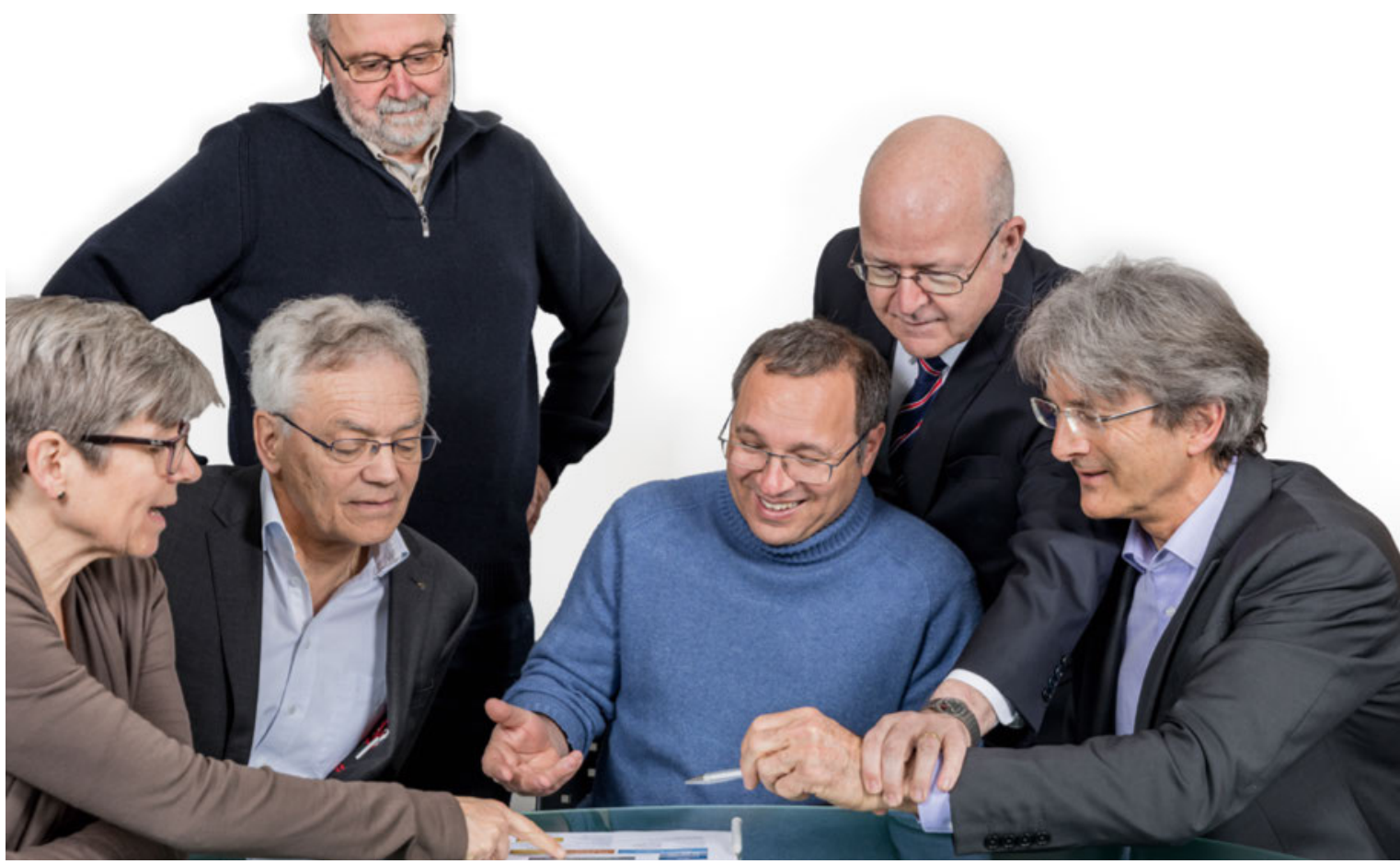

La direction de I'ISFM: Regula Schmid, Giatgen A. Spinas, Jean Pierre Keller, Raphael Stolz, Werner Bauer et Christoph Hänggeli (de gauche à droite). 
sort des nombreux contacts avec l'étranger. Les visites d'établissement par exemple, qui ont lieu après chaque changement de responsable, suscitent un vif intérêt. Par ailleurs, le Département de l'intérieur procède en ce moment à l'accréditation de la formation postgraduée. Tous les sept ans, cela permet de renouveler le mandat de l'ISFM pour l'octroi des titres fédéraux de spécialiste mais c'est aussi l'occasion de formuler des conditions. Selon nos informations, les recommandations des experts sont foncièrement positives.

\section{Le modèle de formation postgraduée suisse associe prestation et enseignement. Cela ne crée-t-il pas des conflits?}

Regula Schmid: Ce modèle jouit d'une longue tradition et remplit sa mission de manière satisfaisante tant que les conditions requises sont données aux établissements de formation: les médecins cadres-doivent suffisamment encadrer et superviser les médecins assistants. Les expériences acquises au lit du patient, au bloc opératoire ou pendant les consultations constituent en effet un fondement crucial pour développer les compétences nécessaires. Sans médecins motivés et sans suffisamment de temps, cela ne fonctionne pas. Werner Bauer: Vous parlez de conflits. Oui, il y en a et ils s'accentuent. Les conditions-cadres de la formation ne s'améliorent pas en raison d'exigences en matière d'efficacité toujours plus élevées dans les hôpitaux, tout comme les charges administratives et économiques des médecins cadres. Dans les hôpitaux aussi, le temps c'est de l'argent. Mais, hélas, le financement de la formation postgraduée est loin d'être garanti de manière satisfaisante. C'est pourquoi nous devons tous faire preuve aujourd'hui d'un engagement particulier, sans perdre de vue que la transmission des compétences nécessaires aux générations futures revêt une importance absolument essentielle.

\section{De nouvelles méthodes didactiques pourraient-elles venir épauler la formation postgraduée et continue?}

Giatgen Spinas: Certainement, mais jusqu'à un certain point. Avec le soutien de simulateurs, il est déjà possible aujourd'hui de proposer de très bonnes formations continues interdisciplinaires en anesthésie, en médecine intensive et aussi en médecine d'urgence. La simulation et la robotique, actuellement en pleine expansion, contribueront aussi à surmonter une diffi- culté croissante, à savoir disposer d'un nombre suffisant de cas pour s'exercer à des interventions chirurgicales.

\section{L'ISFM peut-il influencer le nombre de médecins en formation selon les disciplines?}

Jean Pierre Keller: Cette question fait l'objet de vives discussions, aussi du point de vue de la proportion de médecins de famille et de médecins spécialistes. Les sociétés de discipline et l'ISFM ne peuvent exercer qu'une influence indirecte et limitée sur le nombre de spécialistes via les exigences des programmes de formation. La question de savoir si un pilotage pertinent avec une planification adaptée au besoin est possible et souhaitable suscite plutôt le scepticisme.

\section{L'ISFM a aussi un mandat à honorer dans le domaine} de la formation continue. Le fait-il avec la plateforme de formation continue?

Raphael Stolz: Oui, mais pas seulement. Chaque société de discipline élabore un programme sur la base de la Réglementation pour la formation continue de l'ISFM répondant aux aspects particuliers de la discipline. La plateforme de formation continue est un instrument qui fonctionne sans problème pour documenter la formation continue et avec lequel les sociétés de discipline valident les sessions saisies avant que le diplôme de formation continue puisse être imprimé. Même si la médecine est une profession académique qui s'exerce de manière autonome et responsable, nous ne pouvons pas ignorer que la formation continue est un devoir et que les patients, les assureurs et les politiques attendent qu'il soit rempli et attesté de manière crédible.

\section{Comment voyez-vous les prochains développements?} On parle déjà d'un continuum de la formation prégraduée, postgraduée et continue. Qu'en est-il? Werner Bauer: Ce continuum devient de plus en plus important et c'est un immense défi de le mettre en place. Demain, le champ d'action d'un médecin au moment de partir à la retraite sera très différent de celui d'un médecin qui vient d'obtenir son titre de spécialiste. Le maintien des compétences et l'apprentissage de nouvelles compétences exigeront la création de structures et d'évaluations supplémentaires. $\mathrm{Au}$ niveau mondial, on ne parle d'ailleurs pratiquement plus de formation continue mais de "continuing professional development». Vu sous cet angle, l'ISFM ne devrait pas être à court de travail.

Crédit photo:

Tobias Schmid/ISFM 